CLINICAL STUDY

\title{
Normal overall mortality rate in Addison's disease, but young patients are at risk of premature death
}

\author{
Martina M Erichsen ${ }^{1}$, Kristian Løvås ${ }^{1,2}$, Kristian J Fougner ${ }^{3}$, Johan Svartberg ${ }^{4,5}$, \\ Erik R Hauge $^{6}$, Jens Bollerslev ${ }^{7,8}$, Jens P Berg ${ }^{8,9,10}$, Bjarne Mella ${ }^{11}$ and Eystein S Husebye ${ }^{1,2}$ \\ ${ }^{1}$ Section for Endocrinology, Department of Medicine, Haukeland University Hospital, N-5021 Bergen, Norway, ${ }^{2}$ Institute of Medicine, University of \\ Bergen, N-5007 Bergen, Norway, ${ }^{3}$ St. Olavs University Hospital, N-7030 Trondheim, Norway, ${ }^{4}$ Institute of Clinical Medicine, University of Tromso, \\ N-9037 Tromsø, Norway, ${ }^{5}$ Department of Medicine, University Hospital of North Norway, 9038 Trømsø, Norway, ${ }^{6}$ NKS Olaviken Hospital for Old Age \\ Psychiatry, N-5009 Bergen, Norway, ${ }^{7}$ Rikshospitalet-Radiumhospitalet University Hospital, N-0310 Oslo, Norway, ${ }^{8}$ University of Oslo, Oslo, Norway, \\ ${ }^{9}$ Ullevål University Hospital, N-0450 Oslo, Norway, ${ }^{10}$ Aker University Hospital, N-0514 Oslo, Norway and ${ }^{11}$ Østfold Hospital, N-1606 Fredrikstad, \\ Norway
}

(Correspondence should be addressed to M M Erichsen; Email: mmer@helse-bergen.no)

\begin{abstract}
Context: Primary adrenal insufficiency (Addison's disease) is a rare autoimmune disease. Until recently, life expectancy in Addison's disease patients was considered normal.

Objective: To determine the mortality rate in Addison's disease patients.

Design and methods: i) Patients registered with Addison's disease in Norway during 1943-2005 were identified through search in hospital diagnosis registries. Scrutiny of the medical records provided diagnostic accuracy and age at diagnosis. ii) The patients who had died were identified from the National Directory of Residents. iii) Background mortality data were obtained from Statistics Norway, and standard mortality rate (SMR) calculated. iv) Death diagnoses were obtained from the Norwegian Death Cause Registry.

Results: Totally 811 patients with Addison's disease were identified, of whom 147 were deceased. Overall SMR was 1.15 (95\% confidence intervals (CI) 0.96-1.35), similar in females (1.18 (0.92-1.44)) and males (1.10 (0.80-1.39)). Patients diagnosed before the age of 40 had significantly elevated SMR at 1.50 (95\% CI 1.09-2.01), most pronounced in males (2.03 (1.19-2.86)). Acute adrenal failure was a major cause of death; infection and sudden death were more common than in the general population. The mean ages at death for females (75.7 years) and males (64.8 years) were 3.2 and 11.2 years less than the estimated life expectancy.

Conclusion: Addison's disease is still a potentially lethal condition, with excess mortality in acute adrenal failure, infection, and sudden death in patients diagnosed at young age. Otherwise, the prognosis is excellent for patients with Addison's disease.
\end{abstract}

European Journal of Endocrinology 160 233-237

\section{Introduction}

Primary adrenocortical insufficiency (Addison's disease) is caused by destruction of the adrenal cortex. The disease is rare, with prevalence estimates of 100-140 per million and incidence rates of 5-6 per million per year (1-3), in industrialized countries mostly attributed to autoimmunity $(4,5)$. Autoimmune Addison's disease may occur isolated or as a part of an autoimmune polyendocrine syndrome (APS) (6). About 50\% of patients with Addison's disease have concurrent autoimmune thyroid disease and/or type 1 diabetes mellitus, which is defined as APS type 2 (APS-2). Women are often more afflicted than men, and typically diagnosed between 20 and 50 years of age $(4,7)$. Addison's disease that commences in childhood and adolescence indicates heritable diseases such as APS type 1 (APS-1), which is an autosomal recessive disease caused by defective autoimmune regulator (AIRE) genes $(8,9)$. Other causes are tuberculosis, AIDS, malignant disease, and hereditary diseases such as adrenoleukodystrophy, and a number of rare diseases such as mutations in the dosage-sensitive sex-reversal adrenal hypoplasia congenital gene on the X-chromosome 1 (DAX-1) $(5,10)$.

Once Addison's disease is diagnosed and treated, life expectancy has been considered normal. However, fatal adrenal crises are encountered in clinical practice; the incidence of such causes of deaths is not known. Analyses of the Swedish National Hospital Registry indicated increased mortality in Addison's disease patients, which was mainly ascribed to cardiovascular, malignant, and infectious diseases (11). Excess mortality was also demonstrated in patients with 
hypopituitarism, predominantly from vascular and respiratory disease (12). Lifelong glucocorticoid replacement therapy may cause harm, since most patients receive supra-physiological doses that can possibly institute cardiovascular disease, metabolic disturbances, infections or cancer (13). In autoimmune polyglandular syndromes, excess mortality might also be expected due to concomitant autoimmune disease.

As part of a survey of patients with verified Addison's disease in Norway, we undertook to elucidate if Addison's disease was associated with increased mortality.

\section{Methods}

\section{Overall, study design}

All patients with Addison's disease in Norway were identified in 2005 through a retrospective search in electronic hospital diagnosis registries of in-patients and out-patients. The university hospital first started listing patients in 1972 in an administrative electronic data program. All the hospitals had electronic registration of diagnoses that at least cover the last 15 years. The diagnosis of primary adrenal insufficiency was verified by scrutiny of patient records by endocrinologists.

Deceased patients were identified in the National Directory of Residents; their death causes were retrieved from the National Death Cause Registry. The study was approved by the local ethical committee and the Data Inspectorate of Norway. The study design is illustrated in Fig. 1.

\section{Subjects and diagnosis}

We aimed at identifying all Addison patients in Norway who had been registered in a somatic hospital at least once during the last 15-35 years by searching the in-patient and out-patient registries of all somatic

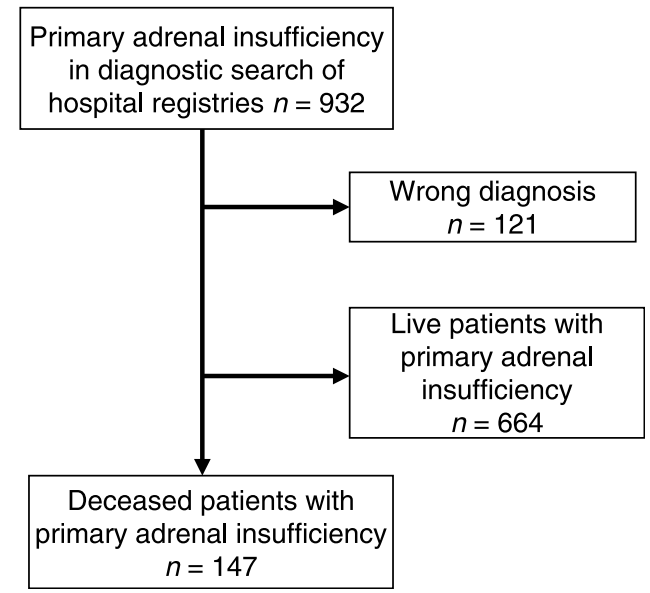

Figure 1 Study design. hospitals in Norway. The common approach with suspected or confirmed autoimmune adrenal insufficiency in Norway is to refer the patient to a consultant endocrinologist in a hospital; thus the patients are likely to be found in the hospital registries. Addison's patients are regularly seen at least once a year by a physician at the internal medicine or endocrinology department at the hospitals. This practice renders their diagnosis easily found in updated hospital registers. The search terminated on December 31st 2005; the medical departments of 57 somatic hospitals contributed lists of possible primary adrenal insufficiency, according to a specified list of diagnostic codes. The patients were identified using the International Classification of Diseases (ICD) codes 255 (ICD 8 and 9: primary adrenal insufficiency, other adrenal disease) and E27.1-4 (ICD 10: primary adrenal insufficiency, adrenal crisis, and other adrenal disease). The codes comprising adrenal tuberculosis, congenital adrenal disorders, and Waterhouse-Friderichsen syndrome were included in the search, but identified no patients with Addison's disease. Patients with adrenal insufficiency after adrenalectomy, adrenoleukodystrophy or congenital adrenal hyperplasia were not included.

The year of diagnosis and the age at diagnosis were taken from the medical records if available. The diagnosis of Addison's disease was confirmed based on the results of basal hormone measurements (simultaneous plasma levels of ACTH above, and serum cortisol below the reference ranges), and/or a pathological ACTH-stimulation test (post-stimulatory serum cortisol levels below $500 \mathrm{nmol} / \mathrm{l}$ ). In older cases, the diagnosis was based on description of clinical signs and symptoms such as hyperpigmentation, salt craving, hypotension, general weakness, weight loss, and typical electrolyte disturbances. Five cases with persisting clinical signs typical for Addison's disease (hyperpigmentation and salt craving), and regular consultations with an endocrinologist were included, based on chronic treatment with glucocorticoids and fludrocortisone. The inclusion of patients in this study was conservative in the sense that cases with insufficient diagnostic information supporting the diagnosis were excluded.

The tenability of this approach has previously been shown in an epidemiological study in Western Norway (14).

\section{Causes of death}

Deceased individuals on our list of patients with Addison's disease were identified in the National Directory of Residents. The Death Cause Registry provided the causes of death using the patient's unique personal identification number. The principal cause of death is the first diagnosis written on the death certificate. The death causes were grouped as death from acute or chronic adrenal failure, cardiovascular 
disease, cancer, infection, sudden death (includes suicide and accidents), diabetes, lung disease, kidney failure, and gastrointestinal disease. The categories were compared with statistics obtained from the general population (Statistics Norway; URL: http://www.ssb.no/ english/).

\section{Data analysis}

Standardized mortality ratio (SMR) was calculated as a standardized incidence ratio for death for the total group to obtain death risk calculation from all observed person-years. Only patients with a known year of diagnosis were used in the SMR calculation. Life expectancy was calculated from Norwegian reference data according to the year of diagnosis and their birthyear cohort; lost or gained years were calculated as the difference between the actual death age and the expected death age. Data are given with 95\% confidence intervals (CI), considered significantly different statistically from normal if the CI does not contain the normative value.

\section{Results}

\section{Patients and diagnostic accuracy}

Altogether, Addison's disease was verified in 811 patients; between 6 and 24 new patients were diagnosed per year over the last 20 years, yielding incidence rates 2.0-6.5 per million per year. One hundred and fortyseven (81 women, 66 men) of the patients had died in the period 1966-2005. The age at diagnosis of the deceased patients was found in the majority of cases $(n=132$; Fig. 2). The mean age at diagnosis for the deceased men was 46 years and for the women, it was 53 years. Five out of the 147 (one female) had APS-1; three of them were not recognized as such in life. One patient with a DAX-1 mutation died of adrenal crisis provoked by an infection at 52 years of age. Six individuals ( 4 males) were diagnosed post-mortem.

Diagnostic accuracy for Addison's disease in live and dead patients was poor; at a university hospital (19872005, ICD 9 and ICD 10) only 38 of 58 patients

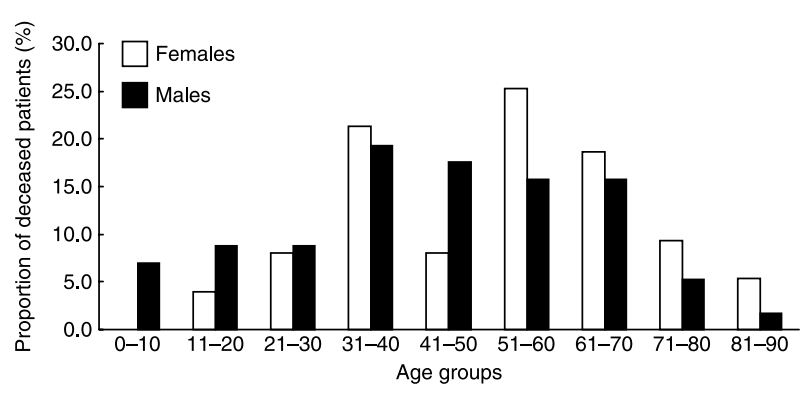

Figure 2 Age at diagnosis for deceased Addison patients. Males, black bars; females, white bars. were coded correctly, i.e. only $66 \%$ had true primary adrenal insufficiency. At another university hospital (2002-2005) the diagnostic accuracy was 60\% (76 of 127 patients) using only the more precise ICD 10 coding. Most of the erroneously coded patients had secondary adrenal failure, due to pituitary disease, adrenalectomy or pharmacological glucocorticoid therapy. Quite a few were patients who had undergone ACTH stimulation test with a negative result. Only those correctly coded were included for further analyses.

\section{Mortality}

Of the 147 dead patients, the age at diagnosis was missing in 15; they were omitted from the calculation of SMR. Overall, the SMR was 1.15 (95\% CI (0.95-1.34)), as calculated from 132 actual and 114 expected deaths. The SMR for females was $1.18(0.92-1.44)$ and for males 1.10 (0.80-1.39). SMR was increased to 1.50 (1.092.01) in patients who had been diagnosed before the age of 40. As illustrated in Fig. 3, this was most pronounced in males, with SMR 2.03 (1.19-2.86), accounting for 23 of 55 deceased male patients. The excess mortality in female patients diagnosed at such young age was not as evident, with SMR 1.23 (0.70-1.76).

The mean death ages for female and male patients were 75.7 and 64.8 years respectively, which is 3.2 and 11.2 years less than the estimated life expectancy at the
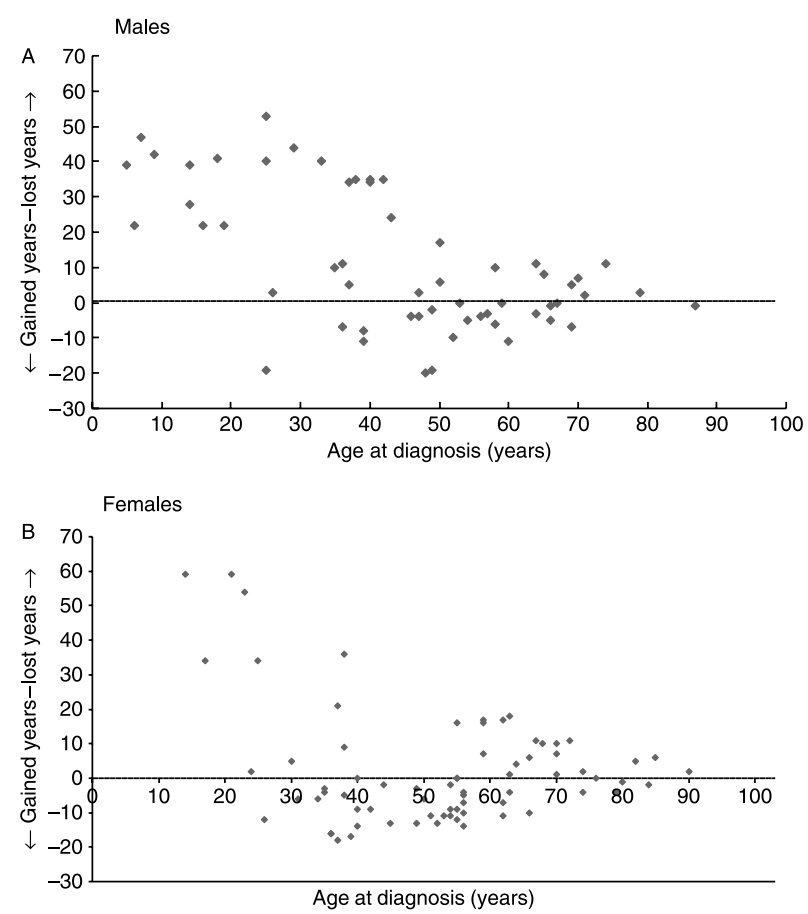

Figure 3 Years lost or gained in relation to the age at diagnosis of Addison's disease. Years lost are given as positive values and years gained as negative values. The dots situated on or close to the $X$-axis illustrate individuals living approximately their life expectancy at diagnosis. A) males, $n=57$; B) females, $n=75$. 
time of diagnosis. Sixty patients outlived their expected age and eight patients lived exactly as long as expected at the time of diagnosis. Sixty-four patients lived shorter than expected from the population statistics.

\section{Causes of death}

The main causes of death are shown in Fig. 4. Adrenal failure was the principal death diagnosis in $15 \%$ of the patients. Infection was the death cause in 10\% (5.114.9 ), opposed to $6.0 \%$ in the general population. Sudden death was found in $9.2 \%$ (4.5-13.9) compared with $5.3 \%$ in the general population. Cardiovascular disease was the most common cause of death accounting for $39 \%$ (31-47), which is comparable to the general Norwegian population during this period (1976-1980: 49\%, 2001-2003: 38\%). Cancer accounted for $13 \%(7.2-18.8)$ of deaths, which is significantly less than in the general population. The distribution of death causes in the general population has, however, changed much over the years, rendering the comparison between the patients and the normal indecisive; for instance during the years 1971-2003, cancer death rates ranged from 19 to $26 \%$.

There was no statistically significant excess mortality due to concomitant endocrine diseases other than diabetes mellitus; principal death diagnosis was diabetes in $4.6 \%(1.2-8.0)$ of our patients as opposed to 1.0$1.5 \%$ in the general population.

\section{Discussion}

Notwithstanding the diagnostic advances and available replacement therapy with cortisone acetate and fludrocortisone, Addison's disease is still a potentially lethal condition. This is clearly illustrated here by the excess mortality in young patients due to acute adrenal insufficiency, sudden deaths, and most likely infections.

Most of the deaths from acute adrenal insufficiency in this study occurred in patients with conventional replacement therapy; in many instances, death was associated with trivial infections. Most likely, there is a potential for patient education, although 98\% of Norwegian patients reported that they have been

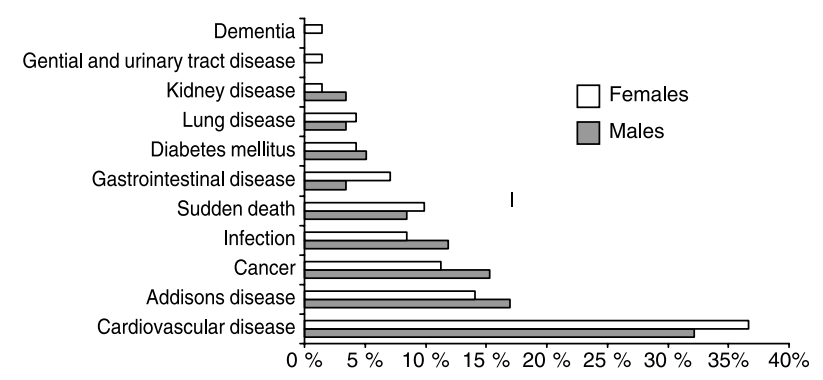

Figure 4 Causes of death in 71 female and 59 male patients with Addison's disease. advised to increase cortisone doses during infections (Erichsen, Norwegian survey of Addison's disease, unpublished data). Patients with Addison's disease and type 1 diabetes mellitus possibly have increased death risk; adrenal crisis is typically complicated by hypoglycemia in these patients. Bergthorsdottir and co-workers found that the overall impact of concomitant diabetes on the excess mortality is minimal (11). The contribution from diabetes to death in our study was $4.6 \%$ (6 patients), which could not be classified further as the older ICD 9 and ICD 8 codes are not differentiating between type 1 and type 2 diabetes.

At the time of diagnosis, and before the start of treatment for Addison's disease, the patients can be critically ill with increased risk of death (15-18). The compensating mechanisms of young people are more prone to keep them circulatory stable until they eventually collapse with a crisis; older patients may call for medical assistance at an earlier stage. One might speculate that young individuals are particularly ignorant to alarm symptoms, but most likely older people with adrenal insufficiency also die undiagnosed. We identified six patients who were diagnosed with adrenal insufficiency post-mortem, which is probably an underestimation. In 1965, Mason et al. reported only two deaths during a five-year follow-up of 74 known patients, but they found 10 new deaths from Addison's disease not diagnosed in life (19). Sudden deaths are not commonly found in hospital records; these deaths are investigated by police authorities and recorded correctly in the Death Cause Registry only if a medico-legal autopsy is performed.

For the whole group of patients, our study shows that the prognosis is good. Patients with Addison's disease are seen regularly by their doctors, who may diagnose and treat other potentially lethal diseases. The other main causes of death in our cohort, that is, cardiovascular disease and cancer, were equally or less frequent than that found in the general population. Thus, our results contrast the analyses of the Swedish National Hospital registry that showed a mortality rate twice as high as normal $(11,20)$. The recent analysis by Bensing and co-workers, however, showed that the cancer rates remained increased only in APS-1 patients, when tumors observed during the first year of follow-up were excluded. In the APS-2 group, most likely to represent true autoimmune Addison's disease, cancer incidence rates were not increased (20).

Norway and Sweden have comparable health care systems that point to study design as the cause of differences in results. The strength of the Swedish study is the number of observed patient years, whereas the comparative strength of our study is the verification of the diagnosis in all the patients and their true age at diagnosis. We included patients only after verification of the diagnosis by an endocrinologist reading the medical records. This quality check revealed that the coding was surprisingly erroneous, which renders pure registry 
studies at jeopardy. Bergthorsdottir and co-workers report less than $8.3 \%$ coding error in the Swedish National Hospital Registry (11), but these numbers represent random quality testing of various diagnoses (21), and may not apply to rare diseases such as Addison's disease. In our experience, adrenal failure is particularly associated with erroneous coding since there are several forms (primary, secondary, iatrogenic, congenital adrenal hyperplasia, and other) that are easily mixed up. Our study also revealed that some patients who were assessed with suspicion of adrenal insufficiency were coded as such even if subsequent work-up turned out negative, causing much of the miscoding error. Thus, scrutiny of medical records of every patient was crucial for obtaining high quality data in Norway. Bergthorsdottir and co-workers performed a quality check of diagnoses that revealed coding error only of $5-6 \%$ at one Swedish hospital. However, the age distribution of that sub-population ( 37 years) was much lower than in the total population identified in the registry (53 years), indicating that the results of the quality check might not apply to the whole population.

Furthermore, the Swedish study by Bergthorsdottir et al. set the first appearance in a hospital registry after 1987 as the age at diagnosis; we verified the true age at diagnosis from the medical records. We believe that the SMR calculation is more correct when all the years from diagnosis until death or censure are taken into account. We suggest that the Swedish study overestimated SMR due to too liberal inclusion of patients at higher age who possibly were miscoded as adrenal insufficiency.

In conclusion, Addison's disease that presented before the age of 40 was associated with excess mortality due to acute adrenal failure, infections, and sudden death, particularly in men. Improved patient education and doctor alertness are important to reduce these untimely fatalities. Otherwise, the prognosis is excellent for patients with Addison's disease.

\section{Declaration of interest}

The authors declare that there is no conflict of interest that could be perceived as prejudicing the impartiality of the research reported.

\section{Funding}

This study was supported by grants from The Western Norway Regional Health Authority to MME who is a doctoral fellow at the institution.

\section{References}

1 Kong MF \& Jeffcoate W. Eighty-six cases of Addison's disease. Clinical Endocrinology $1994 \mathbf{4 1} 757-761$.

2 Laureti S, Vecchi L, Santeusanio F \& Falorni A. Is the prevalence of Addison's disease underestimated? Journal of Clinical Endocrinology and Metabolism $1999 \mathbf{8 4} 1762$.
3 Lovas K \& Husebye ES. High prevalence and increasing incidence of Addison's disease in western Norway. Clinical Endocrinology $200256787-791$.

4 Myhre AG, Undlien DE, Lovas K, Uhlving S, Nedrebo BG, Fougner KJ, Trovik T, Sorheim JI \& Husebye ES. Autoimmune adrenocortical failure in Norway autoantibodies and human leukocyte antigen class II associations related to clinical features. Journal of Clinical Endocrinology and Metabolism 200287 618-623.

5 Betterle C, Dal Pra C, Mantero F \& Zanchetta R. Autoimmune adrenal insufficiency and autoimmune polyendocrine syndromes: autoantibodies, autoantigens, and their applicability in diagnosis and disease prediction. Endocrine Reviews 200223 327-364.

6 Eisenbarth GS \& Gottlieb PA. Autoimmune polyendocrine syndromes. New England Journal of Medicine $2004 \mathbf{3 5 0}$ 2068-2079.

7 Nerup J. Addison's disease - clinical studies. A report of 108 cases. Acta Endocrinologica 197476 127-141.

8 Perheentupa J. Autoimmune polyendocrinopathy-candidiasisectodermal dystrophy. Journal of Clinical Endocrinology and Metabolism 200691 2843-2850.

9 Wolff AS, Erichsen MM, Meager A, Magitta NF, Myhre AG, Bollerslev J, Fougner KJ, Lima K, Knappskog PM \& Husebye ES. Autoimmune polyendocrine syndrome type 1 in Norway: phenotypic variation, autoantibodies, and novel mutations in the autoimmune regulator gene. Journal of Clinical Endocrinology and Metabolism 200792 595-603.

10 Arlt W \& Allolio B. Adrenal insufficiency. Lancet 2003361 1881-1893.

11 Bergthorsdottir R, Leonsson-Zachrisson M, Oden A \& Johannsson G. Premature mortality in patients with Addison's disease: a population-based study. Journal of Clinical Endocrinology and Metabolism $2006914849-4853$.

12 Tomlinson JW, Holden N, Hills RK, Wheatley K, Clayton RN, Bates AS, Sheppard MC \& Stewart PM. Association between premature mortality and hypopituitarism. West Midlands Prospective Hypopituitary Study Group. Lancet 2001357 425-431.

13 Filipsson H, Monson JP, Koltowska-Haggstrom M, Mattsson A \& Johannsson G. The impact of glucocorticoid replacement regimens on metabolic outcome and comorbidity in hypopituitary patients. Journal of Clinical Endocrinology and Metabolism 200691 3954-3961.

14 Løvås K \& Husebye ES. High incidence and increasing prevalence of Addison's disease, an epidemiological study in western Norway. Clinical Endocrinology 200256 787-791.

15 al Sabri AM, Smith N \& Busuttil A. Sudden death due to autoimmune Addison's disease in a 12-year-old girl. International Journal of Legal Medicine 1997110 278-280.

16 Burke MP \& Opeskin K. Adrenocortical insufficiency. American Journal of Forensic Medicine and Pathology 199920 60-65.

17 Keljo DJ \& Squires RH Jr. Clinical problem-solving. Just in time. New England Journal of Medicine 1996334 46-48.

18 Brosnan CM \& Gowing NF. Addison's disease. BMJ 1996312 1085-1087.

19 Mason AS, Meade TW, Lee JA \& Morris JN. Epidemiological and clinical picture of Addison's disease. Lancet $19682744-747$.

20 Bensing S, Brandt L, Tabaroj F, Sjöberg O, Nilsson B, Ekbom A, Blomqvist P \& Kämpe O. Increased death risk and altered cancer incidence pattern in patients with isolated or combined autoimmune primary adrenocortical insufficiency. Clinical Endocrinology $200869697-704$.

21 The National Board of Health and Welfare Socialstyrelsen Dödsorsaker 2002. Causes of death 2002, p 21, Stocköholm: Sveriges officiella Statistik 2004.

Received 3 November 2008

Accepted 3 November 2008 